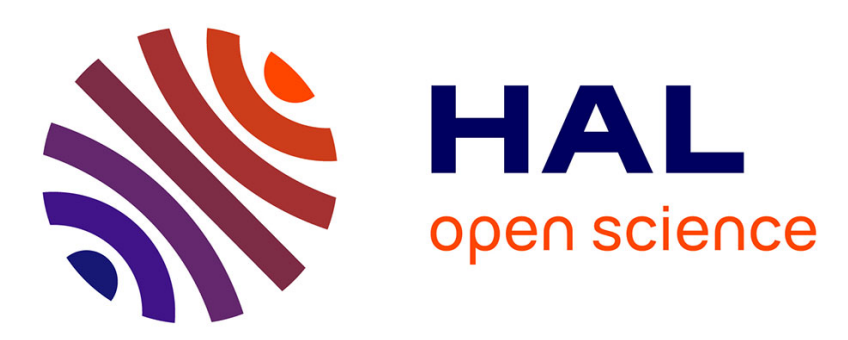

\title{
Dispositif expérimental d'étude de la croissance cristalline à partir de bains fondus \\ M. Gautherie
}

\section{To cite this version:}

M. Gautherie. Dispositif expérimental d'étude de la croissance cristalline à partir de bains fondus. Revue de Physique Appliquée, 1968, 3 (2), pp.131-142. 10.1051/rphysap:0196800302013100 . jpa00242837

\section{HAL Id: jpa-00242837 https://hal.science/jpa-00242837}

Submitted on 1 Jan 1968

HAL is a multi-disciplinary open access archive for the deposit and dissemination of scientific research documents, whether they are published or not. The documents may come from teaching and research institutions in France or abroad, or from public or private research centers.
L'archive ouverte pluridisciplinaire HAL, est destinée au dépôt et à la diffusion de documents scientifiques de niveau recherche, publiés ou non, émanant des établissements d'enseignement et de recherche français ou étrangers, des laboratoires publics ou privés. 


\title{
DISPOSITIF EXPÉRIMENTAL D'ÉTUDE DE LA GROISSANGE GRISTALLINE A PARTIR DE BAINS FONDUS
}

\author{
Par M. GAUTHERIE, \\ Laboratoire de Minéralogie, Faculté des Sciences, Strasbourg. \\ (Reçu le 9 décembre 196\%.)
}

\begin{abstract}
Résumé. - Un appareil a été réalisé pour déterminer par thermométrie infrarouge la distribution des températures à la surface d'un système cristal-bain fondu, et utilisé pour la mesure du facteur d'émission normale, de la conductibilité thermique et des vitesses de croissance et de décroissance de l'étain, du bismuth et du plomb à l'état liquide et à l'état cristallin en fonction de l'orientation cristallographique.
\end{abstract}

Abstract. - An apparatus has been designed for the determination by infrared thermometry of the temperature distribution on the surface of a crystal-melt system, and this has been applied to measurements of normal emissivity, thermal conductivity and growth and melting rates of tin, bismuth and lead in liquid state and in crystalline state versus the crystallographic orientation.

Introduction. - La connaissance de la distribution des températures au voisinage d'un cristal en voie de croissance ou de décroissance en présence du bain fondu est nécessaire pour comprendre les mécanismes de la solidification ou de la fusion, car elle permet de préciser comment s'effectue le transfert de chaleur au sein du système cristal-bain fondu.

En vue d'une telle étude, j'ai réalisé un appareil dont le principe consiste à déterminer la température en mesurant, au moyen d'un détecteur infrarouge, l'énergie émise par un élément de la surface du système cristal-bain fondu. Un dispositif de balayage optique permet d'analyser la température suivant une direction normale à l'interface cristal-bain fondu.

La partie essentielle de l'appareil est le détecteur infrarouge dont le choix détermine la zone des températures mesurables, et par conséquent les corps susceptibles d'être étudiés. Pour des raisons pratiques, j'ai choisi comme détecteur une cellule photorésistante au sulfure de plomb dont le domaine spectral de sensibilité s'étend jusqu'à $2 \mu$, ce qui permet l'étude de phénomènes à des températures supérieures à $200^{\circ} \mathrm{C}$ et m'a conduit à étudier la solidification et la fusion de l'étain, du bismuth et du plomb.

Pour obtenir une image infrarouge utilisable, il fallait éviter, d'une part, les phénomènes de convection et, d'autre part, l'oxydation qui provoque des variations locales du facteur d'émission de la surface, ce

(1) Cet article représente l'essentiel du mémoire de Thèse présenté par M. Gautherie à la Faculté des Sciences de Strasbourg, le 3 juin 1967, pour l'obtention du diplôme de docteur-ingénieur. que j'ai réalisé en plaçant l'ensemble de l'appareil sous vide (environ $10^{-4} \mathrm{~mm} \mathrm{Hg}$ ). Dans ces conditions, l'appareil permet de mettre en évidence des différences de températures de l'ordre de $0,5^{\circ} \mathrm{C}$ sur une surface métallique dont la température est comprise entre $200{ }^{\circ} \mathrm{C}$ et $350^{\circ} \mathrm{C}$.

Pour pouvoir effectuer des mesures absolues de températures, il fallait connaître le facteur d'émission des surfaces étudiées. La comparaison, au moyen du même appareil, des luminances de la surface métallique et d'un corps noir a permis de mesurer le facteur d'émission normale de l'étain, du bismuth et du plomb à leur température de fusion, à l'état liquide et à l'état cristallin en fonction de l'orientation cristallographique.

Lorsque les conditions du transfert de chaleur au sein du système cristal-bain fondu sont telles qu'un état stationnaire où il y a équilibre entre le cristal et le bain fondu est réalisé, il n'y a ni dégagement ni absorption de chaleur latente de changement d'état à l'interface solide-liquide. Dans ces conditions, la distribution des températures au voisinage de l'interface ne dépend que de la conductibilité thermique du cristal et de celle du bain fondu. J'ai pu ainsi déduire de la mesure du rapport des gradients de températures à l'interface le rapport des conductibilités thermiques de l'étain, du bismuth et du plomb à leur température de fusion, à l'état liquide et à l'état cristallin en fonction de l'orientation cristallographique.

Étant donné la différence des facteurs d'émission du cristal et du bain fondu, la courbe de distribution des températures présente une discontinuité qui per- 
met de repérer l'interface solide-liquide et de suivre son déplacement en fonction du temps, et par conséquent de mesurer la vitesse de croissance ou de décroissance. J'ai pu ainsi étudier, en fonction du temps et de l'orientation cristallographique, l'évolution des vitesses de croissance et de décroissance de cristaux de bismuth dans des conditions de températures définies. J'ai comparé les résultats obtenus aux résultats de calculs de transfert de chaleur.

I. Description de l'appareil. - L'appareil comprend (fig. 1) :

A : Four de croissance et décroissance cristalline,

B : Dispositif analyseur optico-mécanique,

C : Dispositif de détection infrarouge.

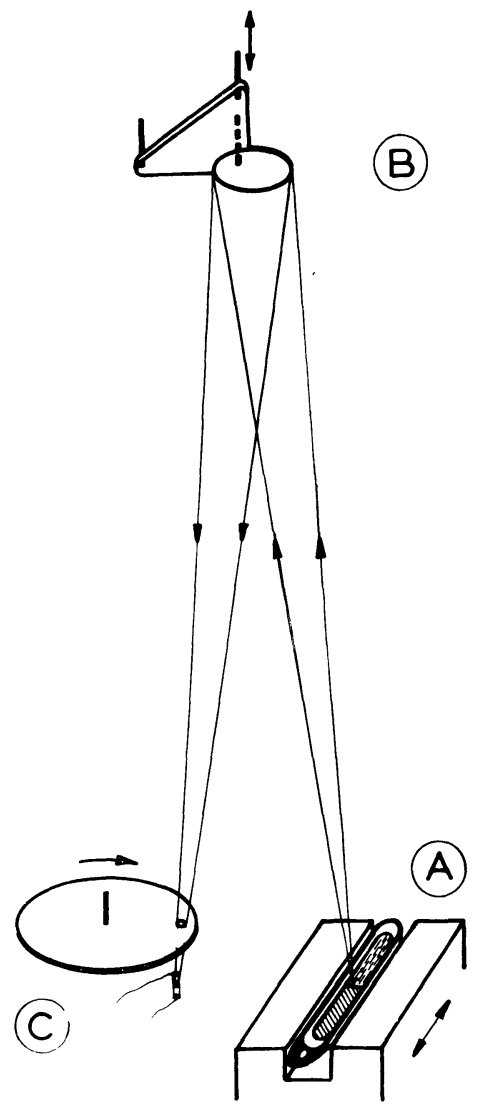

FIG. 1. - Schéma de principe de l'appareil.

L'ensemble est disposé sous vide (environ $10^{-4} \mathrm{~mm} \mathrm{Hg}$ ) dans une enceinte en acier (diamètre : $290 \mathrm{~mm}$, hauteur : $660 \mathrm{~mm}$ ); tous les réglages nécessaires sont effectués avant de faire le vide.

I.1. Four DE GROISSANGE ET DÉGROISSANGE GRISTALLINE ( fig. 2). - La croissance ou la décroissance du cristal a lieu dans une nacelle disposée dans un four ouvert à sa partie supérieure afin de permettre l'observation du rayonnement émis par la surface du

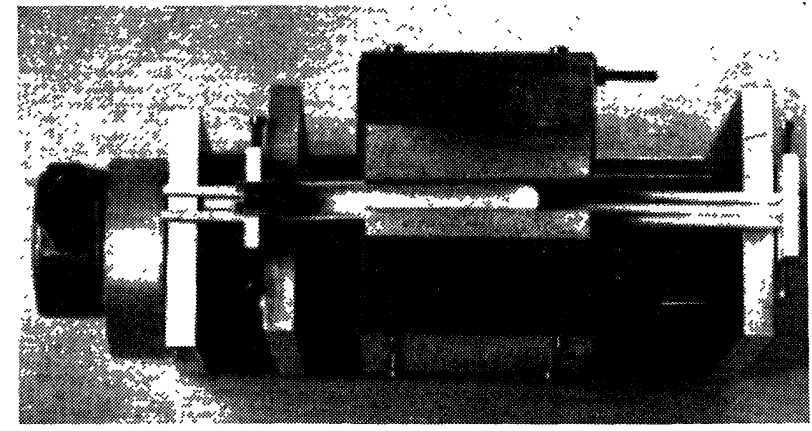

FIG. 2.

Four de croissance et de décroissance cristalline.

système cristal-bain fondu. Il s'agit d'une variante de la méthode du gradient horizontal de Kapitza [1] et Goetz [2].

Le chauffage du four est réalisé par deux enroulements disposés de part et d'autre de la nacelle de façon telle que l'on ait sur un tiers environ de la longueur du four une zone à température à peu près uniforme et sur les deux autres tiers une zone à gradient de température élevé.

La nacelle est fixe, tandis que le four peut se déplacer dans les deux sens, parallèlement à l'axe de la nacelle, grâce à un système écrou-vis sans fin commandé par un moteur synchrone. Cette disposition a été adoptée afin de pouvoir fixer avant chaque expérience les limites de balayage et pour que le bain fondu reste parfaitement immobile.

Le four est entouré d'écrans en tôle d'acier destinés à réduire le rayonnement thermique sur les autres parties de l'appareil.

- Caractéristiques de la nacelle : Matériau : alundum ( $\left.\sim 89 \% \quad \mathrm{Al}_{2} \mathrm{O}_{3}\right)$; Conductibilité thermique : $0,003 \mathrm{cal} / \mathrm{cm}$. s . ${ }^{\circ} \mathrm{C}$; Coefficient de dilatation : $76 \times 10^{-7}$; Dimensions : longueur : $90 \mathrm{~mm}$, largeur et profondeur : $8 \mathrm{~mm}$.

- Caractéristiques du four : Matériau : réfractaire à base d'alumine, moulé en bloc, étuvé à $80^{\circ} \mathrm{C}$, usiné à la main puis cuit au four à $1300^{\circ} \mathrm{C}$ pendant $2 \mathrm{~h}$; Alimentation : tension alternative stabilisée; Vitesse de déplacement dans les deux sens : $8 \mathrm{~mm} / \mathrm{mn}$.

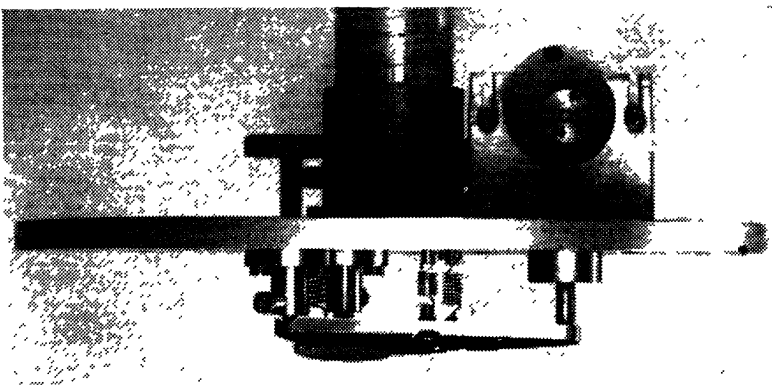

FIG. 3. - Dispositif analyseur optico-mécanique. 
I .2. Dispositif ANAlyseur optico-mÉGANiQUe ( fig. 3). - Ce dispositif a pour but de former l'image d'un point de la surface du système cristal-bain fondu sur la surface sensible ponctiforme du détecteur infrarouge, et de réaliser un balayage rapide suivant la direction de croissance ou de décroissance. Il comporte un miroir concave solidaire d'une plaquette triangulaire maintenue par trois ressorts au contact de trois points grâce à un système trou-trait-plan. Deux des points sont réglables et matérialisent l'axe d'oscillation du miroir perpendiculairement à la direction de croissance ou de décroissance; le troisième est constitué par l'extrémité d'un poussoir commandé par une came tracée de façon à réaliser un balayage proportionnel au temps. La rotation de la came est assurée par un moteur synchrone.

- Caractéristiques du miroir : Diamètre : $34 \mathrm{~mm}$, rayon de courbure : $418,4 \mathrm{~mm}$; Aluminé par vaporisation sous vide.

- Caractéristiques du balayage : Fréquence : $4 \mathrm{~Hz}$; Amplitude à la surface du système cristal-bain fondu : $38 \mathrm{~mm}$.

Ce dispositif, construit sur le principe du levier optique de Chevenard, a été adopté en raison de sa simplicité et de sa facilité de construction.

I.3. Dispositif De DÉtection infrarouge ( $f i g .4$ ). - Il est constitué essentiellement par deux cellules photorésistantes au sulfure de plomb Ektron-Kodak

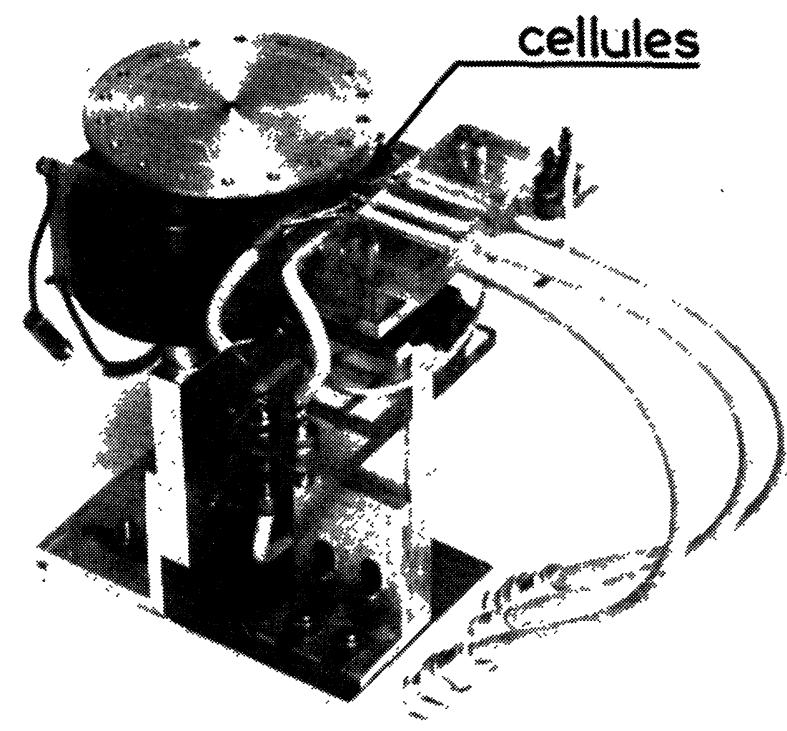

Fig. 4. - Dispositif de détection infrarouge.

type $\mathrm{N}-2$, reliées à un circuit type pont de Wheastone placé à l'extérieur de l'enceinte (liaison par l'intermédiaire de câbles coaxiaux traversant la plaque support de l'enceinte grâce à des passages coaxiaux étanches).

L'une des cellules reçoit le rayonnement émis par la surface du système cristal-bain fondu, modulé à une fréquence de $900 \mathrm{~Hz}$ au moyen d'un disque percé de trous. L'autre permet la compensation du courant d'obscurité et des effets de température.

Les deux cellules sont collées avec un film de picéine sur les faces froides de deux ponts refroidisseurs à effet Peltier et ainsi maintenues à une température à peu près constante d'environ $-20^{\circ} \mathrm{C}$.

Les cellules peuvent être déplacées l'une par rapport à l'autre et sont montées sur un support réglable dans trois directions perpendiculaires afin de permettre l'ajustement de leur surface sensible par rapport aux trous du modulateur.

- Caractéristiques des cellules : Détectivité spécifique à $20^{\circ} \mathrm{C}: D^{*}=9 \times 10^{10} \mathrm{~W}^{-1} \cdot \mathrm{cm} \cdot \mathrm{s}^{-1 / 2}$; Constante de temps à $20^{\circ} \mathrm{C}: 1 \mathrm{~ms}$; Aire de surface sensible : $1 \mathrm{~mm}^{2}$; Résistance d'obscurité à $20^{\circ} \mathrm{C}: 0,18 \mathrm{M} \Omega$.

- Caractéristiques des ponts refroidisseurs : Matériau : poudre agglomérée de tellurure de bismuth soudée à des plaquettes de cuivre constituant les faces « froide » et « chaude »; Alimentation : 0,2 V-6 A par accumulateur au plomb.

L'électronique associée aux cellules au $\mathrm{PbS}$ placée à l'extérieur de l'enceinte comporte successivement : un circuit de polarisation, un amplificateur de tension à faible bruit à transistors Millivac Instr. et un oscilloscope cathodique. Le balayage électronique du tube cathodique est synchronisé avec le balayage optique. L'enveloppe de la porteuse modulée à $900 \mathrm{~Hz}$ représente, aux variations du facteur d'émission près, la distribution des températures à la surface du système cristal-bain fondu suivant la direction de croissance ou de décroissance du cristal.

II. Etude du dispositif de détection infrarouge. II.1. Sensibilité. - Dans le cas de la détermination de la distribution des températures sur une surface donnée, la sensibilité du dispositif de détection infrarouge s'exprime par le plus petit contraste de température décelable entre deux éléments de surface voisins.

J'appelle contraste absolu de luminance $\Delta L^{0}(\lambda)$ la différence entre les luminances monochromatiques de deux corps noirs à des températures $T$ et $T+\Delta T$. La luminance monochromatique $L^{0}(\lambda, T) \mathrm{du}$ corps noir pour une longueur d'onde $\lambda$ et à une température $T$ est donnée par la formule de Planck :

$$
\begin{aligned}
& \quad L^{0}(\lambda, T)=C_{1} \cdot \lambda^{-5} \cdot\left[\exp \left(\frac{C_{2}}{\lambda T}\right)-1\right]^{-1} \\
& C_{1}=1,19 \times 10^{4} \mathrm{~W} \cdot \mathrm{cm}^{-2}, \\
& C_{2}=1,439 \times 10^{4} \mu \cdot{ }^{\mathrm{o}}, \\
& \text { Unités }: \lambda: \mu ; T^{\circ}:{ }^{\circ} ; L^{0}(\lambda, T): \mathrm{W} \cdot \mathrm{sr}^{-1} \cdot \mathrm{cm}^{2} \cdot \mu^{-1} .
\end{aligned}
$$


Le contraste absolu de luminance $\Delta L^{0}(\lambda)$ a pour expression :

$$
\Delta L^{0}(\lambda)=\left[\frac{\partial L^{0}(\lambda, T)}{\partial T}\right] \cdot \Delta T
$$

soit, d'après (1) :

$\Delta L^{0}(\lambda)$

$$
=\frac{C_{1} C_{2}}{T^{2}} \cdot \lambda^{-6} \cdot\left[\exp \left(\frac{C_{2}}{\lambda T}\right)-1\right]^{-2} \cdot \exp \left(\frac{C_{2}}{\lambda T}\right) \cdot \Delta T .
$$

Pour que le dispositif de détection infrarouge ait une bonne sensibilité, le détecteur infrarouge doit avoir un domaine spectral de détectivité centré sur la longueur d'onde $\lambda_{m}^{\prime}$ correspondant au contraste absolu de luminance minimal. La longueur d'onde $\lambda_{m}^{\prime}$ est donnée par une solution de l'équation $\mathrm{d} / \mathrm{d} \lambda\left[\Delta L^{0}(\lambda)\right]=0$, soit :

$$
\lambda_{\mathrm{m}}^{\prime}=\frac{C_{2}}{6} \cdot \frac{1}{T} \simeq \frac{2400}{T}
$$

Unités $: \lambda_{\mathrm{m}}^{\prime}: \mu ; T:{ }^{\mathrm{o}} \mathrm{K}$.

Pour des raisons matérielles, j'ai dû utiliser les cellules photorésistantes au sulfure de plomb, bien que leurs caractéristiques ne soient pas optimales ( fig. 5).

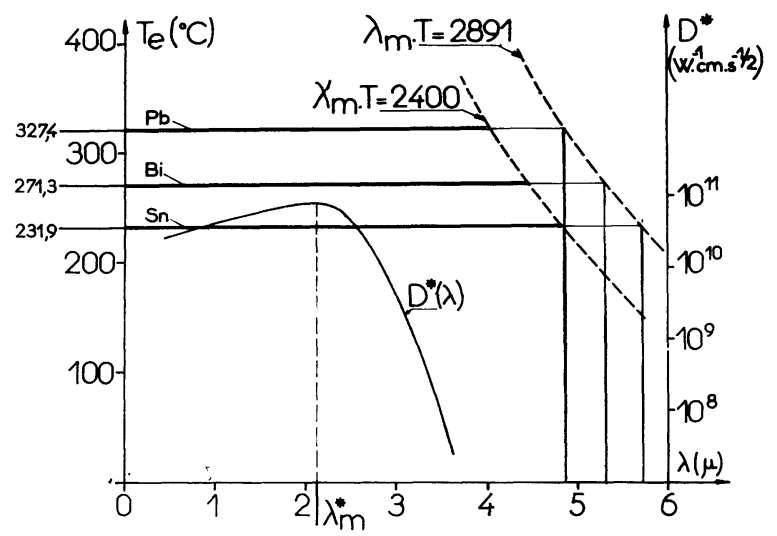

FIG. 5. - Courbe de détectivité spectrale $D^{*}(\lambda)$ des cellules photorésistantes au $\mathrm{PbS}$, Ektron-Kodak type $\mathrm{N}-2$.

- Longueurs d'onde $\lambda_{m}$ correspondant aux maxima de luminance monochromatique du corps noir aux températures de fusion de $\mathrm{Sn}, \mathrm{Bi}$ et $\mathrm{Pb}$ (loi $\lambda_{\mathrm{m}} \cdot T=2891$ ) et longueurs d'onde $\lambda_{m}^{\prime}$ correspondant au contraste absolu de luminance minima à ces mêmes températures (loi $\lambda_{m}^{\prime} \cdot T=2400$ ).

Remarques : 1) Le choix d'un détecteur infrarouge dont le domaine spectral de détectivité soit centré sur la longueur d'onde $\lambda_{\mathrm{m}}^{\prime \prime}$ pour laquelle le contraste relatif de luminance $\Delta L^{0}(\lambda) / L^{0}(\lambda)$ est minimal ne peut être envisagé ici, car la luminance monochromatique au voisinage de $\lambda_{m}^{\prime \prime}$ est beaucoup trop faible.
2) Dans le cas d'une surface métallique dont le facteur d'émission dépend de la longueur d'onde, la courbe de luminance spectrale est déplacée du côté des petites longueurs d'onde par rapport à la courbe relative au corps noir.

La sensibilité dépend de la surface étudiée (voir $\S$ IV.3).

II.2. Définition et Gadence D'information. Pour des raisons de simplicité, le système optique est constitué par un miroir sphérique concave. La surface étudiée et la surface sensible du détecteur se trouvent à une distance du miroir égale à son rayon de courbure (grandissement égal à 1), de façon à ce que les aberrations soient minimales.

J'appelle « point » l'élément de la surface étudiée conjugué de la surface sensible du détecteur. L'ensemble des " points » que l'on peut analyser successivement lors d'une oscillation du miroir constitue une « ligne ».

J'appelle définition du dispositif de détection infrarouge le nombre de " points " contenus dans une " ligne ", et cadence d'information le nombre de « lignes » analysées par seconde.

Des essais réalisés avec une grille fine ont montré que l'on peut discerner environ 50 " points " sur la « ligne » de longueur $38 \mathrm{~mm}$ analysée sur la surface étudiée. Le balayage électronique du tube cathodique étant synchronisé avec les oscillations du miroir, la cadence d'information est égale à $4 / \mathrm{s}$.

Remarque : L'éclairement dans le plan de la cellule est fonction de l'angle solide $\Omega$ défini par le «point » de la surface étudiée et la circonférence du miroir; par suite des oscillations du miroir, $\Omega$ est affecté d'une modulation synchrone des mouvements de balayage $\omega(t)$ donnant naissance à un signal parasite. Le calcul montre que ce signal est largement négligeable par rapport au signal équivalent au bruit.

III. Mesure du facteur d'émission normale de $\mathbf{S n}$, $\mathrm{Bí}$ et $\mathrm{Pb}$ à leur température de fusion, à l'état líquide et à l'état cristallin, en fonction de l'orientation cristallographique. - III.1. FAGTEUR D'ÉMISSION D'UNE SURFAGE. - Définition : On appelle facteur d'émission monochromatique $\varepsilon_{\lambda, T}$ d'une surface, pour une longueur d'onde $\lambda$ et à la température $T$, le rapport de la luminance de cette surface pour la même longueur d'onde et à la même température :

$$
\varepsilon_{\lambda, T}=L_{\lambda, T} / L_{\lambda . T}^{0} .
$$

Le facteur d'émission est en général fonction de la longueur d'onde $\lambda$, de la température $T$ et de la direction d'émission e; il dépend en outre de l'état de la surface.

III.2. Mesure du facteur D'ÉMission D'Une SURFaGe mÉtallique. - Dans ce qui suit, je considère le facteur d'émission dans une direction normale à une surface métallique parfaitement propre et je 
mesure dans le proche infrarouge et à la température de fusion le facteur d'émission $\varepsilon_{\mathrm{s}}$ de la surface solide d'orientation cristallographique connue et le facteur d'émission $\varepsilon_{1}$ de la surface liquide.

La méthode, qui consiste à comparer au flux du rayonnement émis par un corps noir le flux du rayonnement émis par la surface métallique, est la suivante : dans un barreau de graphite sont fraisées deux rainures constituant des corps noirs (rapport profondeur/largeur égal à 6 , d'après les données de Michaud [3]), et une nacelle contenant le métal (fig. 6). Ce barreau est placé dans le four de croissance, d'abord dans la zone à gradient de température élevée pour préparer le cristal dans la nacelle même, et

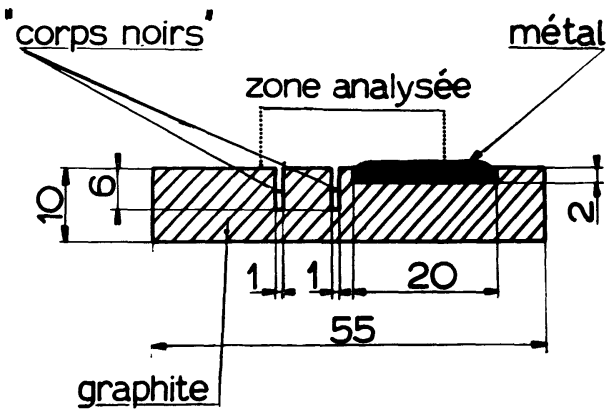

FIG. 6. - Vue en coupe du barreau de graphite permettant la comparaison des flux des rayonnements émis par un corps noir et une surface métallique. ensuite dans la zone à température à peu près uniforme pour les mesures proprement dites.

Le dispositif décrit précédemment permet de tracer sur l'écran d'un oscilloscope une courbe qui représente en première approximation la distribution des facteurs d'émission ( fig. 7); le facteur d'émission de la surface métallique est égal au rapport des amplitudes des signaux correspondant à l'émission respectivement de la surface métallique et du corps noir.

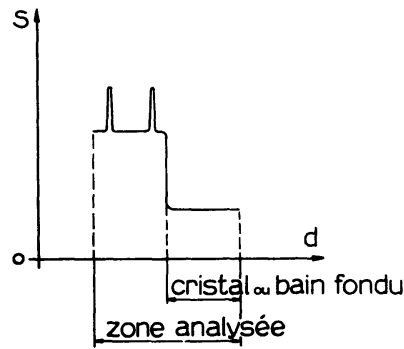

a

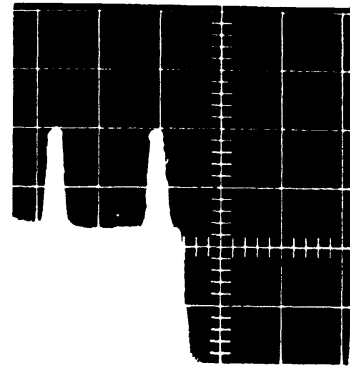

b
FIG. 7. - Courbe de distribution des facteurs d'émission : a) Représentation schématique des variations de l'amplitude du signal de sortie du dispositif de détection infrarouge $S$ en fonction de la distance mesurée suivant l'allongement de la nacelle $d$.

b) Exemple de courbe obtenue dans le cas du plomb liquide.

TABLEAU I

Sn

\begin{tabular}{ccc}
\hline$\varepsilon_{\mathrm{s}}$ & $\alpha$ & $\varepsilon_{1}$ \\
- & - & - \\
0,121 & $88^{\circ}$ & 0,195 \\
0,119 & 85 & 0,192 \\
0,120 & 81 & 0,194 \\
0,119 & 80 & 0,193 \\
0,120 & 79 & 0,190 \\
0,121 & 71 & 0,193 \\
0,122 & 57 & 0,190 \\
0,122 & 51 & 0,190 \\
0,124 & 49 & 0,197 \\
0,130 & 12 & 0,191 \\
0,129 & 9 & 0,187 \\
0,131 & 5 & 0,191 \\
0,130 & 1 & 0,190
\end{tabular}

$\mathrm{Bi}$

\begin{tabular}{ccc}
\hline$\varepsilon_{\mathrm{s}}$ & $\alpha$ & $\varepsilon_{1}$ \\
- & - & - \\
0,331 & 870 & 0,263 \\
0,332 & 86 & 0,264 \\
0,330 & 86 & 0,260 \\
0,322 & 86 & 0,262 \\
0,328 & 84 & 0,273 \\
0,329 & 83 & 0,262 \\
0,332 & 80 & 0,260 \\
0,332 & 79 & 0,264 \\
0,329 & 78 & 0,267 \\
0,330 & 76 & 0,260 \\
0,331 & 74 & 0,265 \\
0,333 & 69 & 0,260 \\
0,332 & 68 & 0,262 \\
0,329 & 64 & \\
0,350 & 42 & \\
0,372 & 16 & \\
0,373 & 13 & \\
0,377 & 11 & \\
0,374 & 9 & \\
0,380 & 3 & \\
0,380 & 3 & \\
0,382 & 1 &
\end{tabular}

$\mathrm{Pb}$

\begin{tabular}{cc}
\hline$\varepsilon_{\mathrm{s}}$ & $\varepsilon_{1}$ \\
- & - \\
0,181 & 0,242 \\
0,176 & 0,244 \\
0,180 & 0,240 \\
0,182 & 0,239 \\
0,179 & 0,242 \\
0,182 & 0,235 \\
0,180 & 0,240 \\
0,180 & 0,241 \\
0,184 & 0,238 \\
0,181 & 0,242 \\
0,182 & 0,245 \\
0,172 & 0,236 \\
0,181 & 0,241 \\
0,186 & 0,240 \\
& 0,241
\end{tabular}


III.3. RÉsultats. - J'ai choisi d'étudier l'étain, le bismuth et le plomb, d'une part car leur pression de vapeur à la température de fusion est suffisamment faible pour permettre des mesures dans un vide de $10^{-4} \mathrm{~mm} \mathrm{Hg}$, et d'autre part car il est possible d'obtenir de gros cristaux. De plus, l'anisotropie dans le cas de l'étain et du bismuth permet d'étudier les variations du facteur d'émission en fonction de l'orientation cristallographique.

Le tableau I donne l'ensemble des valeurs de $\varepsilon_{\mathrm{s}}$ et de $\varepsilon_{1}$ mesurées (il s'agit des résultats bruts des mesures). Dans le cas de l'étain et du bismuth à l'état cristallin, l'orientation du cristal par rapport à la direction d'émission est donnée par l'angle $\alpha$ que fait cette dernière avec l'axe principal du cristal, et déterminée par rayons X dans le cas de l'étain et par clivage dans le cas du bismuth.

\section{TABLEAU II}

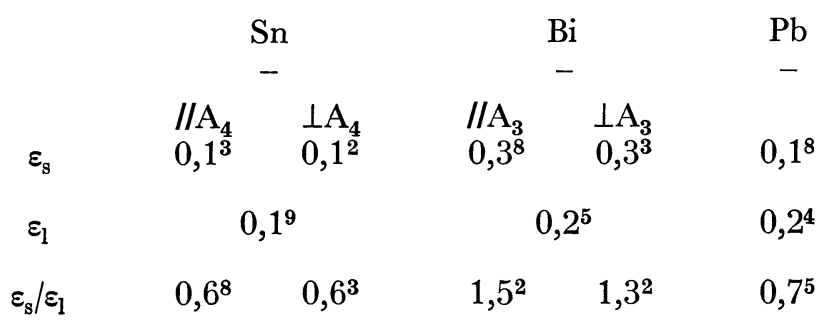

Le tableau II donne les valeurs de $\varepsilon_{\mathrm{s}}$ et de $\varepsilon_{1}$ moyennes calculées d'après les valeurs du tableau I et les valeurs correspondantes du rapport $\varepsilon_{\mathrm{s}} / \varepsilon_{1}$. Dans le cas de l'étain et du bismuth à l'état cristallin, la valeur de $\varepsilon_{\mathrm{s}}$ est donnée pour deux orientations particulières de la direction d'émission, parallèlement $(\alpha=0)$ et perpendiculairement $\left(\alpha=90^{\circ}\right)$ à l'axe principal du cristal.

Compte tenu des écarts moyens relevés ainsi que des conditions expérimentales, l'erreur relative sur la mesure de $\varepsilon_{\mathrm{s}}$ et de $\varepsilon_{\mathrm{l}}$ est estimée inférieure à $5 \%$.

On constate que :

1) Dans le cas de l'étain (quadratique) et du bismuth (rhomboédrique), le facteur d'émission dépend de l'orientation cristallographique et est maximal dans la direction de l'axe principal. Dans le cas du plomb, aucune anisotropie n'a pu être mise en évidence. Dreshfield et House [4] ont mesuré une anisotropie de l'ordre de $0,5 \%$ dans le cas du tantale, du molybdène et du tungstène (cubiques);
2) Lors du changement d'état solide $\rightarrow$ liquide, le facteur d'émission augmente dans le cas de l'étain et du plomb et diminue dans le cas du bismuth.

III.4. Interprétation. - Je désigne par $S_{0}$, $S_{\mathrm{s}}$ et $S_{1}$ les amplitudes des signaux correspondant à l'émission respectivement du corps noir, de la surface solide et de la surface liquide, et par $I, \varepsilon_{\mathrm{s}}$ et $\varepsilon_{1}$ les facteurs d'émission correspondants. Les amplitudes $S_{0}, S_{\mathrm{s}}$ et $S_{\mathrm{l}}$ dépendent essentiellement de la répartition spectrale du rayonnement émis par la surface émettrice (loi de Planck) et de la réponse spectrale de la cellule au $\mathrm{PbS}$ réceptrice, que je représente par une fonction $f(\lambda)$ définie dans l'intervalle $\left(\lambda_{1}, \lambda_{2}\right)$ correspondant au domaine spectral de détectivité de la cellule. Dans ces conditions, l'éclairement énergétique auquel est sensible la cellule peut s'écrire de la manière approchée suivante :

- pour le corps noir :

$$
E^{0}=C_{1} \cdot \int_{\lambda_{1}}^{\lambda_{2}} \lambda^{-5} \cdot\left[\exp \left(C_{2} / \lambda T\right)-1\right]^{-1} \cdot f(\lambda) \cdot \mathrm{d} \lambda
$$

- pour la surface métallique :

$E=C_{1} \cdot \int_{\lambda_{1}}^{\lambda_{2}} \varepsilon(\lambda, T) \cdot \lambda^{-5} \cdot\left[\exp \left(C_{2} / \lambda T\right)-1\right]^{-1} \cdot f(\lambda) \cdot \mathrm{d} \lambda$.

Le facteur d'émission de la surface métallique à la température d'équilibre solide $\rightarrow$ liquide $T=T_{\mathrm{e}}$ a par suite pour expression approchée :

$$
\begin{aligned}
\varepsilon \simeq & \frac{E\left(T_{\mathrm{e}}\right)}{E^{0}\left(T_{\mathrm{e}}\right)} \\
& =\frac{\int_{\lambda_{1}}^{\lambda_{2}} \varepsilon\left(\lambda, T_{\mathrm{e}}\right) \cdot \lambda^{-5} \cdot\left[\exp \left(C_{2} / \lambda T_{\mathrm{e}}\right)-1\right]^{-1} \cdot f(\lambda) \cdot \mathrm{d} \lambda}{\int_{\lambda_{1}}^{\lambda_{2}} \lambda^{-5} \cdot\left[\exp \left(C_{2} / \lambda T_{\mathrm{e}}\right)-1\right]^{-1} \cdot f(\lambda) \cdot \mathrm{d} \lambda} .
\end{aligned}
$$

Les relations entre les propriétés électriques et optiques établies à partir de la théorie électromagnétique de Maxwell et valables pour des longueurs d'onde suffisamment grandes (environ $\lambda>3 \mu$ ) conduisent à l'expression suivante $\mathrm{du}$ facteur d'émission normale monochromatique $\varepsilon_{\lambda, T}$ donnée par Schmidt et Eckert [5] :

$$
\varepsilon_{\lambda, T}=0,365 \cdot\left[r\left(T_{\mathrm{e}}\right)\right]^{1 / 2} \cdot \lambda^{-1 / 2}
$$

$r(T)$ : résistivité électrique à la température $T$ (unités : $\Omega . \mathrm{cm})$,

$\lambda$ : longueur d'onde (unités : $\mathrm{cm}$ ).

Compte tenu de cette dernière relation, l'expression du facteur d'émission s'écrit de la façon suivante :

$$
\varepsilon=0,365 \cdot\left[r\left(T_{\mathrm{e}}\right)\right]^{1 / 2} \cdot \frac{\int_{\lambda_{1}}^{\lambda_{2}} \lambda^{-5,5} \cdot\left[\exp \left(C_{2} / \lambda T_{\mathrm{e}}\right)-1\right]^{-1} \cdot f(\lambda) \cdot \mathrm{d} \lambda}{\int_{\lambda_{1}}^{\lambda_{2}} \lambda^{-5} \cdot\left[\exp \left(C_{2} / \lambda T_{\mathrm{e}}\right)-1\right]^{-1} \cdot f(\lambda) \cdot \mathrm{d} \lambda} .
$$


TABLEAU III

$\begin{array}{cccc} & \mathrm{Sn} & \mathrm{Bi} & \mathrm{Pb} \\ & - & - & - \\ r_{\mathrm{s}}\left(T_{\mathrm{e}}\right)(\Omega . \mathrm{cm}) & 22,5 \times 10^{-6} & 256,5 \times 10^{-6} & 50,5 \times 10^{-6} \\ \varepsilon_{\mathrm{s}} & 0,110 & 0,373 & 0,169 \\ r_{\mathrm{l}}\left(T_{\mathrm{e}}\right)(\Omega . \mathrm{cm}) & 47,5 \times 10^{-6} & 127 \times 10^{-6} & 94,5 \times 10^{-6} \\ \varepsilon_{1} & 0,163 & 0,266 & 0,231 \\ \varepsilon_{\mathrm{s}} / \varepsilon_{1} & 0,67 & 1,40 & 0,74\end{array}$

Le tableau III donne les valeurs de $\varepsilon_{\mathrm{s}}$, $\varepsilon_{1}$ et $\varepsilon_{\mathrm{s}} / \varepsilon_{1}$ calculées d'après la formule (2) et les valeurs de $r\left(T_{\mathrm{e}}\right)$ données par les tables pour le solide polycristallin et le liquide, au moyen d'un ordinateur Bull Gamma Et.

On constate que l'accord entre les valeurs de $\varepsilon_{\mathrm{s}}$ et $\varepsilon_{1}$ mesurées (tableau II) et calculées (tableau III) est satisfaisant; l'écart est dû essentiellement au fait que le domaine spectral de détectivité des cellules au PbS est voisin de la limite d'application de la formule de Drude; l'accord est meilleur en ce qui concerne le rapport $\varepsilon_{\mathrm{s}} / \varepsilon_{1}$ donné par une relation indépendante de la longueur d'onde déduite directement de l'expression (2) :

$$
\left(\frac{\varepsilon_{\mathrm{s}}}{\varepsilon_{\mathrm{l}}}\right)_{T=T_{\mathrm{e}}}=\left[\frac{r_{\mathrm{s}}\left(T_{\mathrm{e}}\right)}{r_{1}\left(T_{\mathrm{e}}\right)}\right]^{1 / 2}
$$

IV. Mesure du rapport des conductibilités thermiques de $\mathrm{Sn}, \mathrm{Bi}$ et $\mathbf{P b}$ à l'état cristallin et à l'état liquide à la température de fusion, en fonction de l'orientation cristallographique. - IV.1. PRINCIPE DE LA MESURE. - La conductibilité thermique est fonction de la température $T$. Dans ce qui suit, je mesure à la température de fusion le rapport de la conductibilité thermique $k_{\mathrm{s}}$ du cristal d'orientation cristallographique connue à la conductibilité thermique $k_{1}$ du bain fondu. La méthode consiste à déterminer le rapport $g_{\mathrm{s}} / g_{1}$ des gradients de température à l'interface cristal-bain fondu mesurés suivant la normale à l'interface lorsqu'il y a équilibre entre le cristal et le bain fondu, c'est-à-dire en l'absence de tout dégagement ou absorption de chaleur latente de changement d'état. En effet, si l'on néglige la variation de volume à la fusion (de l'ordre de $3 \%$ pour les métaux étudiés) et si l'on suppose que le transfert de chaleur au sein du système cristal-bain fondu s'effectue seulement par conduction, le bilan thermique à l'interface à l'équilibre peut s'exprimer par la relation suivante :

$$
k_{\mathrm{s}} \cdot g_{\mathrm{s}}-k_{1} \cdot g_{1}=0 .
$$

Cette hypothèse est justifiée par le fait que l'on opère dans le vide et qu'aux températures de fusion de Sn $\left(231,9^{\circ} \mathrm{C}\right), \mathrm{Bi}\left(271,3^{\circ} \mathrm{C}\right)$ et $\mathrm{Pb}\left(327,4^{\circ} \mathrm{C}\right)$, le transfert de chaleur par rayonnement est relativement négligeable. Cette dernière condition est d'autant mieux réalisée que le flux de chaleur à travers le système cristal-bain fondu suivant la normale à l'interface est plus grand, c'est-à-dire que les conductibilités thermiques $k_{\mathrm{s}}$ et $k_{1}$ et les gradients de températures $g_{\mathrm{s}}$ et $g_{1}$ sont plus grands.

IV . 2. « Modus OPERANDi ». - Le cristal est préparé dans une nacelle en alundum par la méthode du gradient horizontal. Le chauffage du four est ensuite réglé de façon à faire fondre le cristal à partir d'une extrémité de la nacelle à une vitesse de plus en plus faible jusqu'à atteindre un état stationnaire où il y a équilibre entre le cristàl et le bain fondu. Le dispositif de balayage en lumière infrarouge permet alors de déterminer la distribution des températures à la surface du système cristal-bain fondu suivant la direction longitudinale de la nacelle. La courbe obtenue sur l'écran de l'oscilloscope présente à l'endroit de l'interface une discontinuité due à la différence des facteurs d'émission des deux phases. De part et d'autre de cette discontinuité, la courbe représente en première approximation la distribution des températures. L'allure de la courbe obtenue dépend du sens de la variation du facteur d'émission lors du changement d'état solide $\rightarrow$ liquide (fig. 8 et 9 ).

Si je désigne par :

- $p_{\mathrm{s}}$ et $p_{1}$ les pentes mesurées en volts $/ \mathrm{cm}$ des tangentes à la courbe à l'interface respectivement du côté du solide et du côté du liquide,

- $\sigma_{\mathrm{s}}$ et $\sigma_{1}$ les sensibilités mesurées en volts $/{ }^{\circ} \mathrm{C}$ du dispositif de détection infrarouge à la température de fusion respectivement pour le solide et pour le liquide,

le rapport $g_{s} / g_{1}$ des gradients de températures à l'interface a pour expression :

$$
\frac{g_{1}}{g_{\mathrm{s}}}=\frac{p_{1}}{p_{\mathrm{s}}} \cdot \frac{\sigma_{\mathrm{s}}}{\sigma_{\mathrm{l}}}
$$

Le rapport $p_{\mathrm{s}} \mid p_{1}$ est mesuré sur la courbe obtenue sur l'écran de l'oscilloscope et le rapport $\sigma_{\mathrm{s}} / \sigma_{1}$ est déterminé à partir d'un étalonnage de l'appareil. 


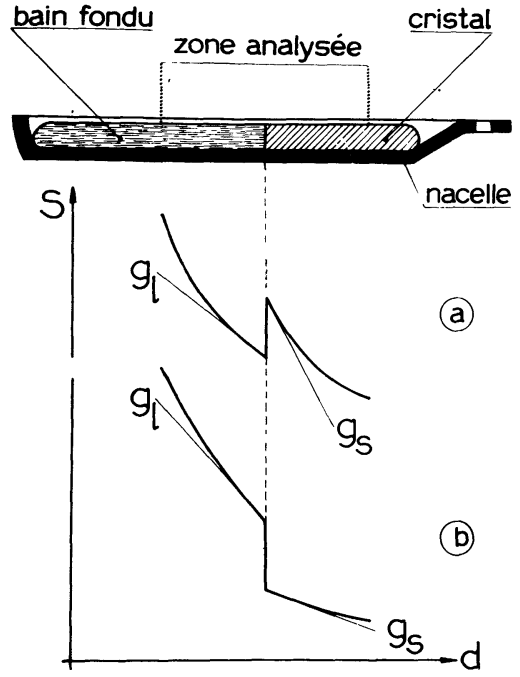

Iig. 8. - Courbe de distribution des températures à l'interface cristal-bain fondu à l'équilibre : représentation schématique des variations $S(d)$.

a) Cas où $\varepsilon_{\mathrm{g}}>\varepsilon_{\mathrm{l}}$, ex. : Bi.

b) Cas où $\varepsilon_{\mathrm{s}}<\varepsilon_{1}$, ex. : Sn, Pb.

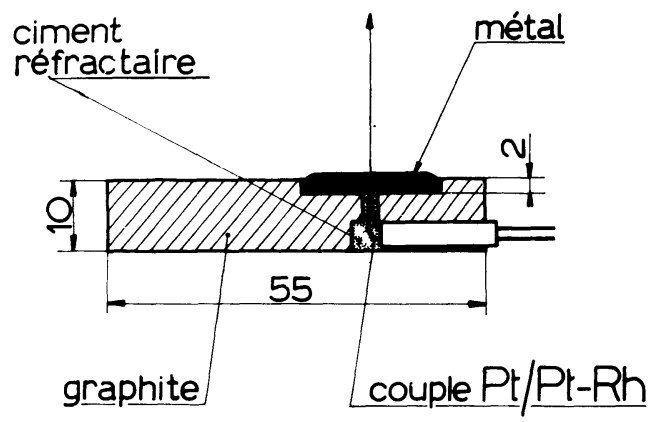

Frg. 9. - Vue en coupe du barreau de graphite utilisé pour l'étalonnage de l'appareil.

IV.3. ÉtAlonNAge DE L'APPAREIL. - Le signal de sortie du dispositif de détection infrarouge $S$ est fonction de la température $T$ et du facteur d'émission $\varepsilon$ de la surface émettrice. Pour une surface donnée, la relation entre $S$ et $T$ dépend d'un grand nombre de paramètres caractéristiques de l'appareil. Étant donné que le facteur d'émission $\varepsilon$ varie avec la température, et ceci de façon différente suivant la nature et l'état du métal, l'étalonnage doit être fait pour chaque surface métallique étudiée à la fois à l'état cristallin et à l'état liquide. Pour effectuer cet étalonnage, j'ai utilisé un barreau de graphite dans lequel est fraisée une nacelle contenant le métal. La température est mesurée au moyen d'un couple thermo-électrique $\mathrm{Pt} / \mathrm{Pt}-\mathrm{Rh}$ dont la soudure chaude est enrobée dans une mince couche de réfractaire durcie par étuvage à $200^{\circ} \mathrm{C}$. De même que pour les mesures de facteur d'émission, le cristal est d'abord préparé dans la nacelle même en plaçant le barreau dans la zone du four à gradient de température élevé. Le miroir concave est réglé de façon à former sur la surface sensible du détecteur infrarouge l'image d'un point de la surface métallique situé juste au-dessus de la soudure chaude du thermocouple ( $f$ ig. 9). Afin de réduire le plus possible l'écart entre la température de la surface émettrice et la température indiquée par le couple, le chauffage du four est réglé de façon telle que la vitesse de chauffe au voisinage de la température d'équilibre solide $\rightarrow$ liquide du métal étudié soit inférieure à $1^{\circ} \mathrm{C} / \mathrm{mn}$.

La mesure simultanée de l'amplitude du signal de sortie du dispositif de détection infrarouge $S$ et de la température $T$ permet de tracer la courbe des variations de $S(T)$ pour $\mathrm{Sn}, \mathrm{Bi}$ et $\mathrm{Pb}$ à l'état liquide, à l'état surfondu et à l'état cristallin. Dans le cas de l'étain et du bismuth à l'état cristallin, les mesures ont été faites avec des cristaux préparés de façon telle que leur axe principal soit perpendiculaire ou parallèle à la direction d'émission (introduction d'un germe orienté dans le bain légèrement surfondu).

On constate qu'en première approximation la sensibilité $\sigma=(\partial S / \partial T)_{T}$ est constante dans un intervalle de température d'environ $20^{\circ} \mathrm{C}$ de part et d'autre de la température d'équilibre solide $\rightarrow$ liquide.

\section{TABLEAU IV}

\begin{tabular}{|c|c|c|c|c|}
\hline$\sigma\left(\mathrm{mV} /{ }^{\circ} \mathrm{C}\right)$ & & & & \\
\hline- & & & & \\
\hline & $/ / \mathrm{A}_{4}$ & $\perp \mathrm{A}_{4}$ & $/ / \mathrm{A}_{3}$ & $\perp A_{3}$ \\
\hline$\sigma_{\mathrm{s}}$ & $0,03^{5}$ & $0,03^{2}$ & 0,27 & 0,23 \\
\hline$\sigma_{1}$ & & & & \\
\hline$\sigma_{\mathrm{s}} / \sigma_{\mathrm{l}}$ & 0,68 & 0,63 & 1,50 & 1,28 \\
\hline
\end{tabular}

Le tableau IV donne les valeurs de $\sigma_{\mathrm{s}}$, $\sigma_{1}$ et $\sigma_{\mathrm{s}} / \sigma_{\mathrm{l}}$ à la température d'équilibre solide $\rightarrow$ liquide calculées d'après les courbes $S(T)$. Compte tenu des écarts moyens relevés sur les courbes $S(T)$ ainsi que des conditions expérimentales, l'erreur relative sur la mesure du rapport $\sigma_{\mathrm{s}} / \sigma_{1}$ est estimée à environ $5 \%$.

Dans le cas particulier de l'étain et du bismuth à l'état cristallin, le rapport $\sigma_{\mathrm{s}} / \sigma_{\mathrm{l}}$ dépend de l'orientation du cristal par rapport à la direction d'émission e. En première approximation, on peut représenter ces variations par un ellipsoïde de révolution autour d'un axe dirigé suivant l'axe principal du cristal. Dans une expérience donnée, connaissant l'orientation du cristal par rapport à e, on détermine la valeur correspondante du rapport $\sigma_{\mathrm{s}} / \sigma_{1}$ en cherchant l'intersection de e et de l'ellipsoïde. 
IV.4. Résultats. - Le tableau V donne les valeurs du rapport $p_{1} / p_{\mathrm{s}}$ mesurées (résultats bruts des mesures) et les valeurs correspondantes du rapport $k_{\mathrm{s}} / k_{1}$ calculées d'après les relations (3) et (4), connaissant le rapport $\sigma_{\mathrm{s}} / \sigma_{1}$. Dans le cas de l'étain et du bismuth à l'état cristallin, l'orientation du cristal par rapport à e est donnée par l'angle $\alpha$ que fait cette dernière avec l'axe principal du cristal et déterminée par rayons $\mathrm{X}$ dans le cas de l'étain et par clivage dans le cas du bismuth.

Il était intéressant de comparer les valeurs du rapport $k_{\mathrm{s}} / k_{1}$ déterminées expérimentalement avec les valeurs de ce rapport relatives au solide polycristallin, d'une part mesurées par Goss [6], et d'autre part calculées d'après l'expression suivante :

$$
k_{\mathrm{s}} / k_{1}=r_{\mathrm{l}} / r_{\mathrm{s}}
$$

déduite de l'équation de Wiedemann-Franz-Lorenz qui relie la résistivité électrique $r$ à la conductibilité thermique $k$ à une température $T$ :

$$
k . r / T=\text { Constante. }
$$

Le tableau VI donne les valeurs du rapport $k_{\mathrm{s}} / k_{1}$ calculées d'après la relation (5) à partir des valeurs de $r$ données par les tables, et les valeurs moyennes du rapport $k_{\mathrm{s}} / k_{1}$ calculées d'après les valeurs mesurées. Dans le cas de l'étain et du bismuth à l'état cristallin, la valeur du rapport $k_{\mathrm{s}} / k_{1}$ est donnée pour deux orientations particulières de la direction d'émission parallèlement ou perpendiculairement à l'axe principal du cristal. Compte tenu des écarts moyens relevés ainsi que des conditions expérimentales, l'erreur relative sur la mesure $\mathrm{du}$ rapport $k_{\mathrm{s}} / k_{\mathrm{l}}$ est estimée inférieure à $7 \%$.

TABLEAU $\mathrm{V}$

ÉTAIN

$\begin{array}{cccc}\alpha & p_{\mathrm{l}} / p_{\mathrm{s}} & \sigma_{\mathrm{s}} / \sigma_{\mathrm{l}} & k_{\mathrm{s}} / k_{\mathrm{l}} \\ - & - & \overline{2} & - \\ 82^{\circ} & 3,32 & 0,634 & 2,07 \\ 80 & 3,32 & 0,635 & 2,08 \\ 78 & 3,31 & 0,635 & 2,07 \\ 74 & 3,24 & 0,636 & 2,03 \\ 71 & 3,20 & 0,638 & 2,01 \\ 68 & 3,07 & 0,639 & 1,93 \\ 67 & 3,10 & 0,639 & 1,95 \\ 64 & 2,98 & 0,640 & 1,88 \\ 63 & 2,94 & 0,642 & 1,86 \\ 58 & 2,84 & 0,644 & 1,80 \\ 54 & 2,76 & 0,650 & 1,77 \\ 22 & 2,27 & 0,674 & 1,53 \\ 18 & 2,24 & 0,678 & 1,52 \\ 12 & 2,21 & 0,679 & 1,50 \\ 2 & 2,16 & 0,680 & 1,47\end{array}$

Bismuth

$\begin{array}{cccc}\alpha & p_{1} / p_{\mathrm{s}} & \sigma_{\mathrm{s}} / \sigma_{1} & k_{\mathrm{s}} / k_{\mathrm{l}} \\ - & - & - & - \\ 86 & 0,45 & 1,28 & 0,58 \\ 79 & 0,44 & 1,28 & 0,56 \\ 78 & 0,44 & 1,28 & 0,56 \\ 76 & 0,44 & 1,28 & 0,56 \\ 69 & 0,42 & 1,29 & 0,54 \\ 66 & 0,41 & 1,29 & 0,53 \\ 59 & 0,40 & 1,30 & 0,52 \\ 53 & 0,38 & 1,33 & 0,51 \\ 51 & 0,37 & 1,34 & 0,49 \\ 32 & 0,32 & 1,42 & 0,45 \\ 29 & 0,29 & 1,43 & 0,42 \\ 18 & 0,29 & 1,47 & 0,43 \\ 13 & 0,30 & 1,48 & 0,44 \\ 5 & 0,29 & 1,50 & 0,44\end{array}$

Plomb

$\begin{array}{ccc}p_{1} / p_{\mathrm{s}} & \sigma_{\mathrm{s}} / \sigma_{1} & k_{\mathrm{s}} / k_{\mathrm{I}} \\ - & - & - \\ 2,50 & 0,74 & 1,85 \\ 2,46 & 0,74 & 1,82 \\ 2,43 & 0,74 & 1,80 \\ 2,47 & 0,74 & 1,83 \\ 2,41 & 0,74 & 1,78 \\ 2,46 & 0,74 & 1,82 \\ 2,45 & 0,74 & 1,81 \\ 2,45 & 0,74 & 1,81 \\ 2,48 & 0,74 & 1,84 \\ 2,42 & 0,74 & 1,79 \\ 2,45 & 0,74 & 1,81 \\ 2,43 & 0,74 & 1,80 \\ 2,42 & 0,74 & 1,79 \\ 2,40 & 0,74 & 1,78\end{array}$

\begin{tabular}{|c|c|c|c|c|c|}
\hline & & n & $\begin{array}{l}\mathrm{Bi} \\
-\end{array}$ & & $\begin{array}{c}\mathrm{Pb} \\
-\end{array}$ \\
\hline \multirow{3}{*}{$k_{\mathrm{s}} / k_{1}$ calculé } & 2,02 & (a) & 0,43 & (a) & $1,88 \quad(\mathrm{a}$ \\
\hline & 2,10 & (b) & 0,45 & (b) & $1,98(d$ \\
\hline & 2,12 & (c) & 0,47 & (c) & $2,06 \quad(\mathrm{c}$ \\
\hline \multirow[t]{2}{*}{$k_{\mathrm{s}} / k_{1}$ mesuré } & 1,8 & (e) & 0,67 & (e) & $1,7 \quad(\mathrm{e}$ \\
\hline & $/ / \mathrm{A}_{4}$ & $\perp \mathrm{A}_{4}$ & $/ / \mathrm{A}_{3}$ & $\perp \mathrm{A}_{3}$ & \\
\hline$k_{\mathrm{s}} / k_{\mathrm{I}}$ mesuré & $1,4^{8}$ & $2,1^{0}$ & $0,4^{4}$ & $0,5^{8}$ & $1,8^{1}$ \\
\hline
\end{tabular}

\section{TABLEAU VI}

(a) : Tsutsumi (1918) ; (b) : Northrup (1914) ; (c) : Vassura (1892) ; (d) : Powell (1955) ; (e) : Goss (1952). 
On constate que :

1) L'accord entre les valeurs mesurées et calculées est satisfaisant;

2) Dans le cas de l'étain (quadratique) et du bismuth (rhomboédrique), la conductibilité thermique dépend de l'orientation cristallographique et est maximale dans une direction perpendiculaire à l'axe principal;

3) Lors du changement d'état solide $\rightarrow$ liquide, la conductibilité thermique diminue dans le cas de l'étain et du plomb et augmente dans le cas du bismuth.

V. Mesure de l'évolution au cours du temps des vitesses de croissance et de décroissance de cristaux de bismuth dans des conditions de températures données. - V.1. Principe DE LA Mesure. - Comme on l'a déjà vu au $\S$ IV.2, la courbe de distribution des températures obtenue sur l'écran de l'oscilloscope présente à l'endroit de l'interface cristal-bain fondu une discontinuité due à la différence des facteurs d'émission des deux phases. La chronophotographie de l'écran de l'oscilloscope pendant la croissance ou la décroissance d'un cristal permet ainsi, d'une part de suivre le déplacement de l'interface et par suite de mesurer la vitesse de croissance ou de décroissance,

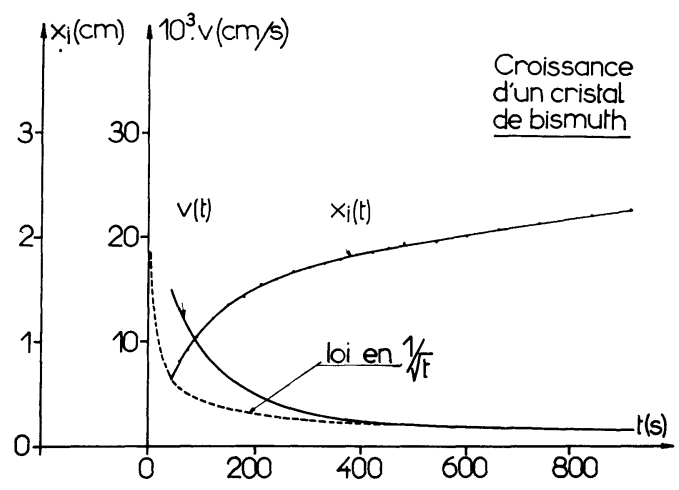

FIG. 10. - Observation de l'interface cristal-bain fondu au moyen du dispositif de balayage en lumière infrarouge émise, dans le cas du bismuth $\left(\varepsilon_{\mathrm{s}}>\varepsilon_{\mathrm{l}}\right)$. Les clichés de l'écran de l'oscilloscope sont pris de $30 \mathrm{~s}$ en $30 \mathrm{~s}$.

et d'autre part de mesurer les températures (fig. 10). Cette méthode est analogue à celle que j'ai déjà utilisée pour mesurer les vitesses de croissance de cristaux d'or [7].

V.2. « Modus Operandi ». - La croissance ou la décroissance du cristal a lieu dans une nacelle en alundum placée dans la zone du four à gradient de température élevé. Le chauffage du four est réglé de façon telle qu'à partir de l'instant initial de la croissance ou de la décroissance les températures des extrémités du cristal et $\mathrm{du}$ bain fondu restent à peu près stationnaires (variations de l'ordre de $0,1^{\circ} \mathrm{C} / \mathrm{mn}$ ). D'après l'enregistrement chronophotographique de la distribution des températures, on trace la courbe $x_{\mathrm{i}}(t)$ des déplacements de l'interface cristal-bain fondu. La dérivée de cette courbe représente la vitesse de croissance ou de décroissance $\left(v=\mathrm{d} x_{\mathrm{i}} / \mathrm{d} t\right)$. On constate (fig. 11) que la vitesse décroît très rapidement, puis

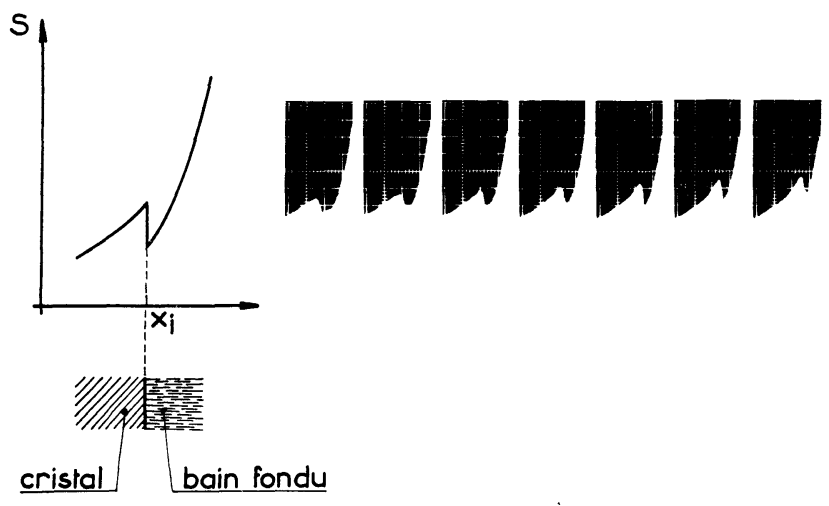

Fig. 11. - Exemple de mesure des déplacements de l'interface cristal-bain fondu au cours du temps : courbe $x_{\mathrm{i}}(t)$, et des vitesses de croissance : courbe $v(t)$.

évolue selon une loi en $1 / \sqrt{ } \bar{t}$ que je représente par les expressions $v=c .1 / \sqrt{t}$ dans le cas de la croissance et $v=d .1 / \sqrt{t}$ dans le cas de la décroissance. J'appelle $c$ et $d$ les coefficients de vitesse.

V.3. INTERPRÉtATION ET RÉSUltats. - Il était intéressant d'essayer de déterminer par le calcul les lois de variations de la vitesse de croissance ou de décroissance dans des conditions voisines des conditions expérimentales. Dans ce but, j'ai fait les hypothèses suivantes :

1) La distribution des températures dans le cristal et le bain fondu suivant la direction de croissance ou de décroissance satisfait à l'équation aux dérivées partielles suivante :

$$
\begin{aligned}
\frac{\partial T(x, t)}{\partial t} & =a^{2} \cdot \frac{\partial^{2} T(x, t)}{\partial x^{2}} \\
a^{2} & =k / C_{\mathrm{p}} \cdot \rho
\end{aligned}
$$

$k$ : conductibilité thermique,

$C_{\mathrm{p}}$ : chaleur spécifique,

$\rho$ : densité.

Cette hypothèse est justifiée par le fait que l'on opère dans le vide à des températures et dans un champ de gradients de températures tels que les pertes de chaleur par convection et rayonnement sont relativement négligeables.

2) La température de l'interface $T_{\mathrm{i}}$ est supposée égale à la température d'équilibre solide $\rightarrow$ liquide $T_{\mathrm{e}}$. Cette hypothèse est justifiée par les observations en 
accord avec le fait que dans le cas des métaux et pour de faibles vitesses de croissance ou de décroissance le degré de déséquilibre thermique à l'interface $\left(T_{\mathrm{e}}-T_{\mathrm{i}}\right)$ est de l'ordre de $0,1^{\circ} \mathrm{C}$.

3) La variation de volume lors du changement d'état est négligée $(\mathrm{Bi}:-2,9 \%)$, de sorte que le bilan thermique à l'interface peut être représenté par l'équation suivante :

$$
\left.k_{\mathrm{s}} \cdot \frac{\partial T_{\mathrm{s}}(x, t)}{\partial x}\right|_{x=x_{\mathrm{i}}}-\left.k_{1} \cdot \frac{\partial T_{1}(x, t)}{\partial x}\right|_{x=x_{\mathrm{i}}}= \pm L \rho v
$$

$L$ : chaleur latente de changement d'état; les indices $\mathrm{s}$ et 1 se rapportent respectivement au cristal et au bain fondu et les signes + et - à la croissance et à la décroissance.

Cette hypothèse est justifiée par les résultats des mesures de conductibilité thermique.

Le calcul opérationnel permet de montrer que la vitesse de déplacement de l'interface varie dans ces conditions selon une loi en $1 / \sqrt{t}$, les coefficients $c$ et $d$ étant donnés par les expressions suivantes :

- dans le cas de la croissance :

$$
\begin{aligned}
& \left(T_{\mathrm{e}}-T_{\mathrm{s}_{\mathrm{o}}}\right) \cdot \frac{k_{\mathrm{s}}}{a_{\mathrm{s}}} \cdot \frac{\exp \left(-c^{2} / a_{\mathrm{s}}^{2}\right)}{\operatorname{erf}\left(c / a_{\mathrm{s}}\right)} \\
& -\left(T_{\mathrm{1}_{\mathrm{o}}}-T_{\mathrm{e}}\right) \cdot \frac{k_{1}}{a_{1}} \cdot \frac{\exp \left(-c^{2} / a_{1}^{2}\right)}{\left[1-\operatorname{erf}\left(c / a_{1}\right)\right]}=\sqrt{\pi} . L . \rho . c
\end{aligned}
$$

— dans le cas de la décroissance :

$$
\begin{aligned}
& \left(T_{1_{\mathrm{o}}}-T_{\mathrm{e}}\right) \cdot \frac{k_{1}}{a_{1}} \cdot \frac{\exp \left(-d^{2} / a_{1}^{2}\right)}{\operatorname{erf}\left(d / a_{1}\right)} \\
& -\left(T_{\mathrm{e}}-T_{\mathrm{s}_{0}}\right) \cdot \frac{k_{\mathrm{s}}}{a_{\mathrm{s}}} \cdot \frac{\exp \left(-d^{2} / a_{\mathrm{s}}^{2}\right)}{\left[1-\operatorname{erf}\left(d / a_{\mathrm{s}}\right)\right]}=\sqrt{\pi} . L . \rho . d
\end{aligned}
$$

$T_{\mathrm{s}_{0}}, T_{1_{0}}$ : températures des extrémités du cristal et

\begin{tabular}{|c|c|c|c|c|c|}
\hline$T_{\mathrm{s}_{0}}\left({ }^{\circ} \mathrm{G}\right)$ & $T_{1_{0}\left({ }^{\circ} \mathrm{C}\right)}$ & $\begin{array}{c}c_{\text {mesuré }} \\
\left(\mathrm{cm} \cdot \mathrm{s}^{-1 / 2}\right) \\
-\end{array}$ & $\begin{array}{c}c_{\text {calculé }} \\
\left(\mathrm{cm} \cdot \mathrm{s}^{-1 / 2}\right) \\
-\end{array}$ & $\underline{\varphi}$ & $\chi$ \\
\hline 259 & 307 & 0,019 & 0,0209 & $0^{\circ}$ & $90^{\circ}$ \\
\hline 249 & 308 & 0,036 & 0,0349 & 0 & 90 \\
\hline 260 & 308 & 0,020 & 0,0212 & 2 & 86 \\
\hline 261 & 301 & 0,022 & 0,0197 & 67 & 39 \\
\hline 260 & 279 & 0,028 & 0,0255 & 68 & 61 \\
\hline 254 & 289 & 0,031 & 0,0296 & 70 & 35 \\
\hline 250 & 292 & 0,035 & 0,0325 & 75 & 72 \\
\hline 236 & 311 & 0,044 & 0,0387 & 76 & 56 \\
\hline 248 & 291 & 0,038 & 0,0344 & 82 & 52 \\
\hline 250 & 281 & 0,040 & 0,0351 & 86 & 47 \\
\hline 259 & 308 & 0,022 & 0,0207 & 88 & 29 \\
\hline 259 & 307 & 0,023 & 0,0209 & 90 & 42 \\
\hline
\end{tabular}
du bain fondu.

Les tableaux VII et VIII donnent respectivement, dans le cas de la croissance et dans le cas de la décrois-

\section{TABLEAU VII}

\section{TABLEAU VIII}

$\begin{array}{cccccc}T_{\mathrm{s}_{0}}\left({ }^{\circ} \mathrm{C}\right) & T_{1_{\mathrm{o}}}\left({ }^{\circ} \mathrm{C}\right) & \begin{array}{c}d_{\text {mesuré }} \\ \left(\mathrm{cm} \cdot \mathrm{s}^{-1 / 2}\right)\end{array} & \begin{array}{c}d_{\text {calculé }} \\ \left(\mathrm{cm} \cdot \mathrm{s}^{-1 / 2}\right)\end{array} & \varphi & \chi \\ 264 & - & - & - & - & - \\ 251 & 314 & 0,086 & 0,0804 & 00^{\circ} & 90 \\ 241 & 310 & 0,066 & 0,0629 & 0 & 90 \\ 265 & 314 & 0,079 & 0,0756 & 5 & 39 \\ 263 & 283 & 0,082 & 0,0777 & 67 & 61 \\ 257 & 292 & 0,045 & 0,0419 & 68 & 35 \\ 242 & 313 & 0,057 & 0,0548 & 70 & 72 \\ 259 & 294 & 0,078 & 0,0751 & 75 & 56 \\ 251 & 299 & 0,060 & 0,0578 & 76 & 52 \\ 255 & 298 & 0,066 & 0,0624 & 82 & 47 \\ 261 & 312 & 0,082 & 0,0619 & 86 & 29 \\ 264 & 314 & 0,085 & 0,0779 & 88 & 42\end{array}$


sance, différentes valeurs de $c, T_{\mathrm{s}_{0}}, T_{\mathrm{I}_{0}}$ et $d, T_{\mathrm{s}_{0}}$ $T_{1}$ mesurées, et les valeurs correspondantes de $c$ et $d$ calculées au moyen d'un ordinateur Bull Gamma Et d'après les expressions (6) et (7); les valeurs de $k$, $C_{\mathrm{p}}, \rho, L$ et $T_{\mathrm{e}}$ ont été prises dans les tables et les valeurs de $T_{\mathrm{s}_{0}}$ et $T_{1_{0}}$ ont été mesurées. Compte tenu des conditions expérimentales et des écarts moyens relevés sur les courbes $x_{\mathrm{i}}(t)$, l'erreur relative sur la mesure des coefficients $c$ et $d$ est estimée à environ $3 \%$. Dans chaque cas, on donne l'orientation du cristal par les angles $\varphi$ et $\chi$ que fait la direction de croissance ou de décroissance avec respectivement la direction [111] (axe ternaire) et la direction [1피. $\varphi$ et $\chi$ sont déterminés sur une projection stéréographique après avoir mesuré au moyen d'un rapporteur les angles que font les clivages (111) et (111) avec la direction de croissance ou de décroissance.

On constate que :

1) L’accord entre les valeurs mesurées et calculées est satisfaisant;

2) Les coefficients de vitesse $c$ sont inférieurs aux coefficients de vitesse $d$, ce qui s'explique par le fait qu'à l'interface cristal-bain fondu se produisent un dégagement de chaleur dans le cas de la croissance et une absorption de chaleur dans le cas de la décroissance;

3) Les coefficients de vitesse $c$ et $d$ dépendent de l'orientation cristallographique ( fig. 12) : dans le cas de la croissance, la chaleur de cristallisation est évacuée par le cristal, et par conséquent $c$ est maximal lorsque la direction de croissance coïncide avec une direction de plus grande conductibilité thermique du cristal, en l'occurrence une perpendiculaire à l'axe ternaire. Dans le cas de la décroissance, la chaleur apportée par le bain fondu est en partie absorbée par la fusion et le reste évacué par le cristal; par conséquent, $d$ est maximal lorsque la direction de décroissance coïncide avec l'axe ternaire, direction de plus

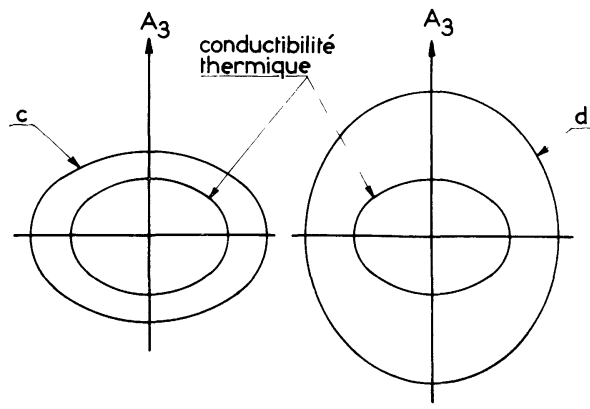

Croissance

Décroissance

FIG. 12. - Représentation schématique des variations des coefficients de vitesse $c$ et $d$ en fonction de l'orientation cristallographique du cristal.

faible conductibilité thermique du cristal. D'autre part, l'influence de l'orientation cristallographique est beaucoup moins marquée sur les coefficients $d$ que sur les coefficients $c$.

- Remarque : Dans ces expériences, les coefficients de vitesse ne dépendent pas directement de la notation cristallographique de l'interface, mais essentiellement du flux de chaleur dans le cristal et le bain fondu. En effet, l'interface reste constamment perpendiculaire à la direction de croissance ou de décroissance, quelle que soit l'orientation cristallographique du cristal.

Remerciements. - Ce travail a été effectué au Laboratoire de Minéralogie de la Faculté des Sciences de Strasbourg sous la direction de M. le Professeur S. Goldsztaub et avec l'aide de la Société UgineKulhmann. Je remercie MM. les Professeurs D. Bernard, G. Boutry, A. Coche et H. Saucier pour leurs précieux conseils et MM. Brissot, Gayzac et Welschinger pour leur aide. J'exprime ma reconnaissance à MM. G. Long et H. de Lacheisserie.

\section{BIBLIOGRAPHIE}

[1] Kapitza (P.), Proc. Roy. Soc. London, 1928, 9, 97.

[2] Goetiz (A.), Phys. Rev., 1961, 35, 462.

[3] Michaud (M.), C. R. Acad. Sci., 1948, 226 B, 999.

[4] DRESHFIELD (R. L.) et House (R. D.), AI A A Journal, $1966,4,371$
[5] Schmidt (E.) et ECKERT (E.), Forsch. a. d. Geb. d. Ingenieurwes., 1935, 1.

[6] Goss (A. J.), Proc. Phys. Soc., 1952, 66, 7 B, 525.

[7] Gautherie (M.), C. R. Acad. Sci., 1966, 262 B, 1078. 